\title{
Padrão endometrial em mulheres submetidas à laqueadura tubária
}

\author{
Endometrial status in women submitted to tubal ligation
}

\begin{abstract}
Maria Letícia Fagundes ${ }^{1}$, Maria Célia Mendes², Maristela Carbol Patta ${ }^{2}$, Marcos Dias de Moura ${ }^{3}$, Antônio Dorival Campos ${ }^{4}$, Geraldo Duarte ${ }^{5}$, Marta Edna Holanda Diógenes Yazlle ${ }^{2}$
\end{abstract}

\section{RESUMO}

Objetivo: estudar as modificações histológicas que ocorrem no endométrio de mulheres antes e seis meses após serem submetidas à laqueadura tubária (LT) e correlacionar esses achados com os níveis de progesterona (P4) sérica. Método: foram incluídas 16 mulheres com ciclos menstruais normais, antes e no sexto ciclo após a LT. Os níveis de P4 foram determinados a partir do $8^{\circ}$ dia, de 2 em 2 dias até a ovulação e no $8^{\circ}, 10^{\circ}$ e $12^{\circ}$ dia pós-ovulatório ou no $24^{\circ}$ dia do ciclo. Realizou-se biópsia endometrial entre o $10^{\circ}$ e $12^{\circ}$ dia pós-ovulatório ou no $24^{\circ}$ dia do ciclo, correlacionando com a $\mathrm{P} 4$. A análise estatística foi realizada com uso do teste não paramétrico de McNemar para avaliação da dosagem hormonal e o teste exato de Fisher para a avaliação histológica do endométrio, sendo considerado estatisticamente significativo $\mathrm{p}<0,05$. Resultados: a média de idade foi 34,1 $\pm 1,3$ anos. O intervalo intermenstrual foi $27,1 \pm 2,6$ dias e a duração do sangramento de 3 a 5 dias, não havendo diferença entre os períodos estudados. Antes da LT, 8/16 (50,0\%) dos casos tinham endométrio secretor compatível com o dia do ciclo, $3 / 16(18,8 \%)$ secretor incompatível e $3 / 16(18,8 \%)$ disfuncional, sugerindo defeito da fase lútea $6 / 16(37,5 \%)$. Na fase pós-LT, $7 / 16(43,8 \%)$ tinham endométrio secretor compatível, 3/16 (18,8\%) secretor incompatível e 4/16 (25,0\%) disfuncional, sugerindo defeito da fase lútea em 7/16 (43,8\%). Em 2/16 (12,5\%) dos casos antes da LT e outros 2/16 (12,5\%) pós-LT não foi possível fazer a avaliação histológica, devido a material insuficiente ou endometrite inespecífica. Na fase lútea pós-LT, os níveis médios da P4 foram significativamente mais baixos nos dias $+8,+10$ e +12 do que na pré-LT, sendo na pré-LT, respectivamente, 15,$1 ; 18,0$ e $20,7 \mathrm{ng} / \mathrm{mL}$ e na pós-LT, 10,$6 ; 8,0$ e $5,4 \mathrm{ng} / \mathrm{mL}$ (p<0,05). Na pré-LT, 5/8 (62,5\%) dos casos com endométrio compatível tinham P4 $\geq 10 \mathrm{ng} / \mathrm{mL}$ e $3 / 8(37,5 \%)$ tinha P4 $<10 \mathrm{ng} / \mathrm{mL}$. Na pós-LT, quando o endométrio foi secretor compatível, a P4 foi $\geq 10 \mathrm{ng} / \mathrm{mL}$ em $4 / 7$ (57,1\%) e P4 < $10 \mathrm{ng} / \mathrm{mL}$ em 3/7 (42,9\%). Essas diferenças não foram significantes $(\mathrm{p}>0,05)$. Conclusão: seis meses pós-LT, não se modificaram o intervalo intermenstrual e a duração do sangramento. A P4 diminuiu durante a fase lútea, embora não tenha interferido na resposta endometrial.

PALAVRAS-CHAVE: Endométrio/anatomia \& histologia; Progesterona; Esterilização tubária

\section{ABSTRACT}

Purpose: to study the histological modifications that occur in the endometrium of women before and six months after tubal ligation (TL) and to correlate these findings with progesterone (P4) levels. Methods: the study was conducted on 16 women with normal menstrual cycles who were evaluated before and in the sixth cycle after TL. P4 levels were determined from the $8^{\text {th }}$ day at 2-day intervals until ovulation and on the $8^{\text {th }}, 10^{\text {th }}$ and $12^{\text {th }}$ day after ovulation or on the $24^{\text {th }}$ day of the cycle. An endometrial biopsy was obtained between the $10^{\text {th }}$ and $12^{\text {th }}$ day after ovulation or on the $24^{\text {th }}$ day of the cycle and a correlation with P4 was determined. Data were analyzed statistically by the nonparametric McNemar test for the evaluation of hormonal determination and by the exact Fisher test for the histological evaluation of the endometrium, with the level of significance set at $\mathrm{p}<0.05$. Results: mean age was $34.1 \pm 1.3$ years. The intermenstrual interval was $27.1 \pm 2.6$ days and the duration of bleeding was 3 to 5 days, with no difference between the studied periods. Before TL, 8/16 (50.0\%) of the cases had a secretory endometrium according to the cycle, $3 / 16(18.8 \%)$ had a secretory endometrium not according to the cycle and $3 / 16(18.8 \%)$ had a dysfunctional endometrium, suggesting a defect in the luteal phase in 6/16 (37.5\%). After TL, 7/16 $(43.8 \%)$ had a secretory endometrium according to the cycle, $3 / 16(18.8 \%)$ a secretory endometrium not according to the cycle and 4/16 (25.0\%) had a dysfunctional endometrium, suggesting a defect in the luteal phase in $7 / 16(43.8 \%)$. In $2 / 16$ $(12.5 \%)$ of the cases before TL and in 2/16 (12.5\%) other cases after TL it was not possible to perform histological evaluation due to insufficient material or unspecfiic endometritis. In the luteal phase after TL, mean P4 levels were significantly lower

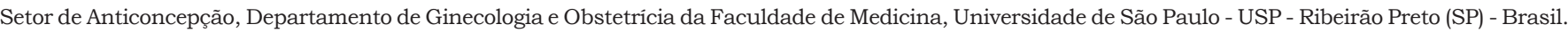
1 Médica Ginecologista do Centro Diagnóstico de Ginecologia e Obstetrícia de Curitiba (PR) - Brasil.

2 Professor Doutor do Departamento de Ginecologia e Obstetrícia da Faculdade de Medicina de Ribeirão Preto-USP

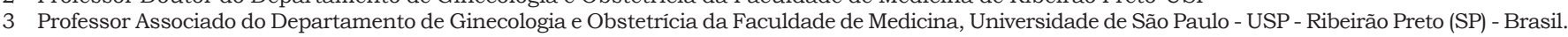

4 Professor Associado do Departamento de Medicina Social da Faculdade de Medicina, Universidade de São Paulo - USP - Ribeirão Preto (SP) - Brasil.

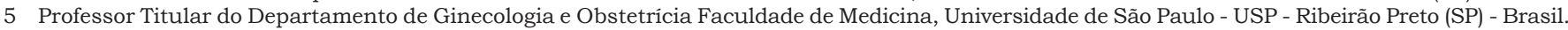

Correspondência: Marta Edna Holanda Diógenes Yazlle

Departamento de Ginecologia e Obstetrícia

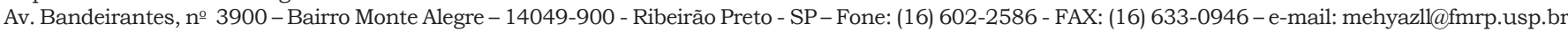


on days $+8,+10$ and +12 than before TL, being $15.1,18.0$ and $20.7 \mathrm{ng} / \mathrm{mL}$, respectively, before TL and $10.6,8.0 \mathrm{and} 5.4 \mathrm{ng} / \mathrm{mL}$ after TL $(\mathrm{p}<0.05)$. Before TL, $5 / 8(62.5 \%)$ of the cases with a secretory endometrium according to the cycle had P4 $\geq 10 \mathrm{ng} / \mathrm{mL}$ and $3 / 8(37.5 \%)$ had P4 $<10 \mathrm{ng} / \mathrm{mL}$. After TL, when the endometrium was secretory according to the cycle, $\mathrm{P} 4 \mathrm{was} \geq 10 \mathrm{ng} / \mathrm{mL}$ in $4 / 7(57.1 \%)$ and $<10 \mathrm{ng} / \mathrm{mL}$ in $3 / 7(42.9 \%)$. These differences were nonsignificant ( $>0.05)$. Conclusion: six months after TL, the intermenstrual interval and the duration of bleeding were unchanged. P4 levels decreased during the luteal phase although this did not interfere in the endometrial response.

KEYWORDS: Endometrium/anatomy \& histology; Progesterone; Sterilization, tubal

\section{Introdução}

A laqueadura tubária (LT) tem sido um dos métodos anticoncepcionais mais utilizados por casais que desejam controlar a fecundidade ${ }^{1}$, provavelmente em função da segurança oferecida e aparente ausência de efeitos colaterais ${ }^{2,3}$.

Alguns estudos fazem referência a possiveis alterações da esteroidogênese ovariana em mulheres submetidas à LT. Essas alterações poderiam estar relacionadas com a técnica utilizada para a oclusão tubária e com o comprometimento da vascularização do mesossalpinge $\mathrm{e}^{4}$, podendo, tardiamente, se refletir no padrão menstrual destas mulheres ${ }^{5}$.

Associação entre esterilização tubária e mudanças no ciclo menstrual pode não ocorrer até 2 anos após a cirurgia ${ }^{6}$. Além disso, a função ovariana e a resistência vascular da artéria ovariana, avaliada pela dopplervelocimetria, também não parecem ser afetadas pelo procedimento ${ }^{7}$. No entanto, alguns autores consideram que a esterilização tubária está associada ao risco aumentado de deficiência de fase lútea ${ }^{8}$.

As características menstruais estão relacionadas às condições endometriais, que por sua vez são decorrentes da ação dos hormônios ovarianos sobre o endométrio. A avaliação histológica do endométrio permite inferir a função ovariana, possibilitando a datação endometrial em um ciclo menstrual clássico de 28 dias $^{9}$. Visto que a esterilização tubária pode determinar alterações hormonais e conseqüentemente levar a modificações no endométrio ${ }^{10}$, seriam esperadas mudanças no padrão de sangramento. Devido a essas considerações, o objetivo deste estudo foi estudar as modificações histológicas que ocorrem no endométrio de mulheres antes e seis meses após serem submetidas à LT e correlacionar esses achados com os niveis de progesterona (P4) sérica nos mesmos períodos da avaliação endometrial.

\section{Métodos}

O estudo incluiu 16 mulheres com indicação para anticoncepção cirúrgica. Todas as pacien- tes assinaram o consentimento informado, aprovado pelo Comitê de Ética do Hospital das Clínicas da Faculdade de Medicina de Ribeirão Preto da Universidade de São Paulo (HCFMRP-USP). Todas foram submetidas, previamente, aos procedimentos preconizados pelo Setor de Anticoncepção do Departamento de Ginecologia e Obstetricia da FMRP-USP, como avaliação clínica geral e ginecológica e coleta de material para colpocitologia oncótica, hemograma e sorologias.

Foram considerados como critérios de inclusão: mulheres com idade entre 30 e 35 anos, ciclos menstruais regulares e fertilidade comprovada com duas ou mais gestações anteriores.

Os critérios de exclusão foram: mulheres em uso de método anticoncepcional, por período mínimo de três meses antes da realização da LT, uso de qualquer medicamento que interferisse nos níveis hormonais, por período minimo de três meses antes da realização da LT, e ausência de qualquer doença que pudesse comprometer o estudo.

O desenho do estudo consistiu no diagnóstico de ovulação feita pela avaliação do muco cervical, ecografia pélvica transvaginal, dosagem da P4 e biópsia endometrial antes e 6 meses após a LT.

A técnica utilizada para oclusão tubária foi a de Pomeroy modificada, por meio de minilaparotomia. Essa técnica consistiu no pinçamento da tuba na porção istmo-ampolar formando-se uma alça apreendida por pinça auxiliar. Com um fio de algodão 2-0, a alça era amarrada e seccionada, bilateralmente.

Para a avaliação do muco cervical foi utilizado o critério de Insler et al. ${ }^{11}$, que consistia na análise do muco cervical aspirado no orificio externo do colo uterino na fase pré-ovulatória, dependendo dos achados ultra-sonográficos. Foram avaliadas as modificações da quantidade, filância e cristalização do muco cervical, além da abertura do orificio externo da cérvice. Os parâmetros recebiam pontuação de 0 a 3 e o seu somatório formava um índice cervical, podendo variar de 0 a12, sendo considerado bom resultado quando a contagem estava acima de 8 .

O acompanhamento ultra-sonográfico foi iniciado a partir do $8^{\circ}$ dia do ciclo menstrual, prosseguindo até o folículo alcançar diâmetros entre 18 e $20 \mathrm{~mm}$, quando em geral rompiam, indicando pos- 
sivel ovulação. Os parâmetros utilizados para a detecção da ovulação foram desaparecimento ou retração do folículo, aumento da ecogenicidade e presença ou não de líquido no fundo de saco de Douglas.

A coleta de sangue para a dosagem de P4 foi realizada na fase lútea do último ciclo menstrual precedente à LT, no $8^{\circ}, 10^{\circ}$ e $12^{\circ}$ dia após a ovulação ou no $20^{\circ}, 22^{\circ}$ e $24^{\circ}$ dia do ciclo menstrual, quando não foi possivel determinar com segurança o dia da ovulação. O mesmo procedimento foi realizado no sexto ciclo menstrual posterior à LT. A dosagem da P4 foi feita pela técnica de radioimunoensaio utilizando kits da DPC (Diagnostic Products Corporation, Los Angeles - USA). Foi considerado ovulação quando os valores da P4 estavam maiores ou iguais a $5 \mathrm{ng} / \mathrm{mL}$. O erro interensaio foi de $10,3 \%$ e o erro intra-ensaio foi de $17,6 \%$, considerados dentro da normalidade.

A biópsia do endométrio foi realizada entre o $10^{\circ}$ e o $12^{\circ}$ dia pós-ovulatório ou no $24^{\circ}$ dia do ciclo menstrual, quando não foi possível determinar com segurança o dia da ovulação. O material coletado por meio da biópsia foi fixado em formol a $10 \%$, processado e analisado no Laboratório de $\mathrm{Pa}-$ tologia e Citologia do HCFMRP-USP, seguindo os critérios clássicos de Noyes et al. ${ }^{9}$. Esse critério baseia-se na avaliação da assincronia no desenvolvimento glandular-estromal e na datação do endométrio, ou seja, na resposta endometrial segundo o dia do ciclo menstrual. Todas as lâminas foram revisadas por um segundo examinador que não tinha conhecimento do dia do ciclo menstrual das mulheres em estudo. Os resultados foram comparados e discutidos e somente os resultados consensuais foram considerados.

Considerou-se insuficiência lútea quando se observava assincronia (ou seja, o desenvolvimento do estroma diferia do desenvolvimento glandular) no desenvolvimento glandular-estromal (endométrio disfuncional) ou quando na datação do endométrio havia defasagem de dois ou mais dias, ou seja, a datação estava adiantada ou atrasada em dois dias em relação ao dia cronológico do ciclo menstrual. Para estabelecer a cronologia do dia do ciclo menstrual, foram considerados o primeiro dia da última menstruação e, principalmente, a data da menstruação subseqüente (avaliação realizada antes e seis meses após a LT). E ainda, foram considerados valores de P4 inferiores de $10 \mathrm{ng} / \mathrm{mL}$ como indicativos de insuficiência lútea, com base nas informações de Johansson ${ }^{12}$ (10 ng/mL como indicativo de ovulação).

A análise estatística foi realizada com o uso do teste não paramétrico de McNemar para avaliação da dosagem hormonal (comparação entre valores hormonais do período pré e pós-LT). O teste exato de Fisher foi utilizado para a avaliação histológica do endométrio (comparação entre a porcentagem de assincronia no endométrio no período pré e pós-LT). Foi considerado estatisticamente significativo $\mathrm{p}<0,05$.

\section{Resultados}

As pacientes tinham idade entre 30 e 35 anos, com média de $34,1 \pm 1,3$ anos. O ciclo menstrual variou de 24 a 32 dias, com média de $27,1 \pm 2,6$ dias, e apresentavam sangramento com duração de 3 a 5 dias. Não houve diferença quanto à idade e à duração do ciclo menstrual e do sangramento entre os períodos pré e pós-LT $(p>0,05)$.

No período pré-LT, o endométrio foi secretor e compatível com o dia do ciclo em 8/16 (50,0\%) dos casos. Em $3 / 16$ (18,8\%) dos casos, o endométrio foi secretor, porém incompatível com o dia do ciclo, e em 3/16 (18,8\%) o achado correspondeu a endométrio disfuncional. Esses resultados foram sugestivos de defeito da fase lútea em $6 / 16(37,5 \%)$ dos casos. Em 2/ 16 (12,5\%) dos casos não foi possivel realizar a avaliação histológica, devido à presença de endometrite inespecífica ou material insuficiente (Tabela 1).

Tabela 1 - Resultados anatomopatológicos de fragmentos endometriais obtidos por biópsia nos períodos pré laqueadura tubária (pré-LT) e pós laqueadura tubária (pós-LT).

\begin{tabular}{lrrrrr}
\hline Histologia do endométrio & \multicolumn{2}{c}{ Pré-LT } & \multicolumn{2}{c}{ Pós-LT } \\
& $\mathbf{n}$ & $\%$ & \multicolumn{1}{c}{$\mathbf{n}$} & $\%$ \\
\hline Endométrio secretor compatível com o dia do ciclo & 8 & 50,0 & 7 & 43,8 \\
Endométrio sugestivo de defeito da fase lútea & 6 & 37,5 & 7 & 43,8 \\
$\quad$ Secretor incompatível com o dia do ciclo & 3 & 18,8 & 3 & 18,8 \\
$\quad$ Disfuncional & 3 & 18,8 & 4 & 25,0 \\
Endométrio não avaliado & 2 & 12,5 & 2 & 12,5 \\
Total & 16 & 100,0 & 16 & 100,0 \\
\hline${ }^{*} p<0,05$. & & & &
\end{tabular}

No período pós-LT, em $7 / 16$ (43,8\%) dos casos observou-se que o endométrio secretor foi compativel com o dia do ciclo. Em 3/16 (18,8\%) o endométrio secretor foi incompativel, enquanto em 4/ $16(25,0 \%)$ o endométrio foi disfuncional, sugerindo defeito da fase lútea em $7 / 16(43,8 \%)$ dos casos. Estes resultados são significativamente diferentes (teste exato de Fisher, $\mathrm{p}<0,05)$. Em 2/ $16(12,5 \%)$ dos casos não foi possivel fazer a avaliação histológica, devido a endometrite inespecífica, ocorrendo em pacientes diferentes daquelas que apresentaram essa mesma patologia no período pré-LT (Tabela 1).

Os valores médios e da mediana da $\mathrm{P} 4$, na fase lútea nos períodos pré-LT e pós-LT, estão apresentados na Tabela 2 , sendo observados níveis significativamente menores nos dias $+8,+10$ e +12 no período pós-LT. No dia +10 , os valores médios 
da $\mathrm{P} 4$ foram 18 e 8,0 $\mathrm{ng} / \mathrm{mL}$, respectivamente, nos períodos pré-LT e pós-LT, e no dia +12 foram 20,7 e $5,4 \mathrm{ng} / \mathrm{mL}$, respectivamente, nos períodos préLT e pós-LT.

Tabela 2 - Níveis médios ( \pm DP) e mediana da progesterona (P4) na fase lútea, nos períodos pré Laqueadura Tubária e pós Laqueadura Tubária.

\begin{tabular}{lrrcc}
\hline Situação/ & \multicolumn{4}{c}{ Dias } \\
parâmetro & $\mathbf{0}$ & $\mathbf{+ 8}$ & $\mathbf{+ 1 0}$ & $\mathbf{+ 1 2}$ \\
\hline Pré-LT Média (ng/mL) & 1,2 & 15,1 & 18,0 & 20,7 \\
$\quad$ DP & $\pm 0,8$ & $\pm 6,6$ & $\pm 8,7$ & $\pm 13,4$ \\
$\quad$ Mediana (ng/mL) & 1,2 & 13,7 & 17,0 & 12,7 \\
Pós-LT Média (ng/mL) & 0,9 & $10,6^{*}$ & $8,0^{* *}$ & $5,4^{\dagger}$ \\
DP & $\pm 0,9$ & $\pm 4,3$ & $\pm 4,2$ & $\pm 3,9$ \\
Mediana (ng/mL) & 0,7 & 11,0 & 8,4 & 4,4 \\
P & 0,37 & 0,03 & 0,003 & 0,04
\end{tabular}

${ }^{*} e^{\dagger} p<0,05$ (comparado ao valor pré $L T$ ); ${ }^{* *} p<0,001$ diferença significante em relação aos valores pré $\mathrm{LT}$.

Foram comparados os resultados de biópsias do endométrio com as dosagens de $\mathrm{P} 4$ no periodo pré-LT e pós-LT, com exceção dos casos em que havia alterações endometriais antes da cirurgia. No período pré-LT, quando o endométrio era secretor compativel com o dia do ciclo, o valor da $\mathrm{P} 4$ era $\geq 10 \mathrm{ng} / \mathrm{mL}$ em $5 / 8(62,5 \%)$ dos casos e $<10$ $\mathrm{ng} / \mathrm{mL}$ em $3 / 8(37,5 \%)$ dos casos. No período pósLT, quando o endométrio era secretor compatível com o dia do ciclo, o valor sérico da P4 era $\geq 10 \mathrm{ng} /$ $\mathrm{mL}$ em $4 / 7$ (57,1\%) dos casos e $<10 \mathrm{ng} / \mathrm{mL}$ em 3/ 7 (42,9\%) dos casos. Essas diferenças não foram significantes $(p>0,05)$.

\section{Discussão}

Os padrões dos distúrbios menstruais em mulheres esterilizadas são sugestivos de sangramentos uterinos disfuncionais semelhantes àqueles causados por defeitos da fase lútea ${ }^{5}$, o que não corresponde aos dados do presente estudo, no qual não foram observadas diferenças no padrão menstrual, quando compararam-se os períodos pré-LT e pós-LT. Esses dados, no entanto, estão compatíveis com os resultados de outros autores, que observaram que a esterilização tubária não causa irregularidade menstrual ${ }^{13}$. Entretanto, observa-se que a esterilização tubária em ratas pode acarretar processo inflamatório endometrial e levar a irregularidade menstrual como complicação precoce ${ }^{14}$.

Os resultados de biópsias endometriais, apresentados na Tabela 1, sugestivos de defeito da fase lútea são semelhantes a outro estudo, em que se observou tendência do endométrio de apresentar risco aumentado de defeito nessa fase, 1 ano após a esterilização tubária ${ }^{8}$. No entanto, utilizando os mesmos critérios, alguns autores ${ }^{15}$ não demonstraram defeitos da fase lútea em mulheres que tinham sido submetidas ao procedimento. Uma provável explicação para a divergência entre os resultados dos estudos acima pode ser a técnica utilizada para o procedimento, pois enquanto alguns utilizaram a técnica cirúrgica clássica, outros empregaram a eletrocoagulação laparoscópica.

O diagnóstico de defeito da fase lútea geralmente é feito por meio do estudo de repetidas biópsias endometriais ${ }^{16}$. Todavia, no presente estudo, foi realizada uma única biópsia nos periodos pré e pós-LT e isto pode ter contribuído ao aparecimento de maior número de defeito nessa fase.

Diferentes pesquisadores têm procurado demonstrar quais os valores de P4 são indicativos de defeito da fase lútea. Um único valor da $\mathrm{P} 4$ de $10 \mathrm{ng} / \mathrm{mL}$ pode ser considerado como indicativo de função normal ${ }^{12}$, ao passo que o somatório dos valores da P4 nos dias 20, 22 e 24 do ciclo menstrual, inferior a $15 \mathrm{ng} / \mathrm{mL}$, pode ser considerado defeito da fase lútea ${ }^{17}$.

No estudo em questão, a análise dos valores de P4 na fase lútea mostrou diminuição no período pós-LT, na maioria das mulheres, embora em alguns casos o valor de P4 tenha se mantido acima de $10 \mathrm{ng} / \mathrm{mL}$, o que indica ovulação ${ }^{18}$.

A diminuição da P4 na fase lútea ocorre por deficiência de produção do corpo lúteo. Sabe-se que o aporte sanguíneo é de fundamental importância para que a função lútea seja adequada, uma vez que no período periovulatório ocorre intensa vascularização das camadas da granulosa e da teca, por aumento da produção local de VEGF, com potente ação vasogênica ${ }^{19}$.

Embora alguns pesquisadores não tenham encontrado alterações nos valores de P4 após a esterilização ${ }^{20}$, é provável que isso ocorra por modificação da vascularização, ocasionada pelo procedimento cirúrgico. Deve ser considerada a possibilidade da ocorrência de distúrbios isquêmicos transitórios, por interferência de suprimento sanguíneo ovariano por lesão da mesossalpinge ${ }^{21}$. No entanto, não foi encontrada diferença no fluxo da artéria uterina e ovariana após a LT, avaliado por dopplervelocimetria transvaginal ${ }^{22}$. Mesmo assim, parece prudente orientar a utilização de técnicas que lesem a menor quantidade de tecido, com a finalidade de preservar a função ovariana ${ }^{23}$. Utilizando a eletrocoagulação para LT, não foram encontradas aceleração do declínio da reserva ovariana e nem disfunção ovariana, cinco anos após o procedimento $^{24}$ ou aos seis meses após a cirurgia ${ }^{25}$.

Niveis de $\mathrm{P} 4 \geq 10 \mathrm{ng} / \mathrm{mL}$, geralmente, determinam padrão endometrial secretor. Esse fato foi verificado nesta pesquisa, na qual as pacientes com esses niveis de P4 apresentavam endométrio secretor compativel com o dia do ciclo em $62,5 \%$ 
antes da cirurgia e 57,1\% após a cirurgia. Quando os niveis de $\mathrm{P} 4$ eram inferiores a $10 \mathrm{ng} / \mathrm{mL}$, foi observado endométrio secretor compativel com o ciclo em $37,5 \%$ no período pré-LT, o que se elevou para $42,9 \%$ no periodo pós-LT, embora sem significado estatístico, ou seja, mesmo com niveis de $\mathrm{P} 4$ inferiores a $10 \mathrm{ng} / \mathrm{mL}$ houve resposta endometrial satisfatória com padrão secretor. As observações acima descritas podem ser explicadas por ocorrer transformação secretória endometrial mesmo com niveis de $\mathrm{P} 4$ inferiores a $3 \mathrm{ng} / \mathrm{mL}^{17,18}$.

É importante enfatizar que os resultados aqui apresentados expressam tendência comportamental do fenômeno avaliado no presente estudo e que, embora seja dificil, trabalhos com maior número de pacientes devem ser realizados para a obtenção de resultados mais definitivos. Além disso, como esse tema desperta grande interesse, é importante o desenvolvimento de novos projetos nessa área.

Concluindo, no presente estudo não foram observadas, seis meses após a LT, modificações no intervalo intermenstrual e nem na duração do sangramento. Os níveis de $\mathrm{P} 4$ diminuíram durante a fase lútea, embora não tenham interferido na resposta endometrial dessas mulheres.

\section{Referências}

1. Baill IC, Cullins VE, Pati S. Counseling issues in tubal sterilization. Am Fam Physician. 2003;67(6):1287-94.

2. Peterson HB, Jeng G, Folger SG, Hillis SA, Marchbanks PA, Wicox LS, et al. The risk of menstrual abnormalities after tubal sterilization. U.S. Collaborative Review of Sterilization Working Group. N Engl J Med. 2000;343(23):1681-7.

3. Nelson DB, Sammel MD, Freeman EW, Gracia CR, Liu L, Langan E. Tubal ligation does not affect hormonal changes during the early menopausal transition. Contraception. 2005;71(2):104-10.

4. Kaiser R, Kusche M, Wurz H. Hormone levels in women after hysterectomy. Arch Gynecol Obstet. 1989;244(3):169-73.

5. Rosenfeld BL, Taskin O, Kafkashli A, Rosenfeld ML, Chuong CJ. Sequelae of postpartum sterilization. Arch Gynecol Obstet. 1998;261(4):183-7.

6. Pati S, Cullins V. Female sterilization. Evidence. Obstet Gynecol Clin North Am. 2000;27(4):859-99.

7. Yazici G, Arslan M, Pata O, Oz U, Aban M. Ovarian function and vascular resistance after tubal sterilization. J Reprod Med. 2004;49(5):379-83.

8. Sumiala S, Tuominen J, Irjala K, Klemi P, Maenpaa $\mathrm{J}$. Luteal function declines after laparoscopic sterilization by Hulka or Filshie clips. Contraception. 2000;62(4):177-80.

9. Noyes RW, Hertig AT, Rock J. Dating the endometrial biopsy. Fertil Steril. 1950;1(1):3-25.
10.Verco CJ, Carati CJ, Gannon BJ. Human endometrial perfusion after tubal occlusion. Hum Reprod. 1998;13(2):445-9.

11.Insler V, Melmed H, Eichenbrenner I, Serr DM, Lunenfeld B. The cervical score a simple semiquantitative method for monitoring of the menstrual cycle. Int $\mathrm{J}$ Gynaecol Obstet. 1972;10(3):223-8.

12.Johansson ED. Progesterone levels in peripheral plasma during the luteal phase of the normal human menstrual cycle measured by a rapid competitive protein binding technique. Acta Endocrinol (Copenh). 1969;61(4):592-606.

13. Shobeiri MJ, Atashkhoii S. The risk of menstrual abnormalities after tubal sterilization: a case control study. BMC Womens Health. 2005;5(1):5.

14. Duran B, Demirkoprulu N, Guvenal T, Arici S, Tuncer E, Cetin M, et al. Histopathological changes in ovary and endometrium after tubal ligation: a rat model. Acta Obstet Gynecol Scand. 2003;82(3):220-4.

15. Hague WE, Maier DB, Schmidt CL, Randolph JF. An evaluation of late luteal phase endometrium in women requesting reversal of tubal ligation. Obstet Gynecol. 1987;69(6):926-8.

16. McNeely MJ, Soules MR. The diagnosis of luteal phase deficiency: a critical review. Fertil Steril. 1988;50(1):1-15.

17. Abraham GE, Maroulis GB, Marshall JR. Evaluation of ovulation and corpus luteum function using measurements of plasma progesterone. Obstet Gynecol. 1974;44(4):522-5.

18. Israel R, Mishell DR Jr, Stone SC, Thorneycroft IH, Moyer DL. Single luteal phase serum progesterone assay as an indicator of ovulation. Am J Obstet Gynecol. 1972;112(8):1043-6.

19. Gordon JD, Shifren JL, Foulk RA, Taylor RN, Jaffe $\mathrm{RB}$. Angiogenesis in human female reproductive tract. Obstet Gynecol Surv. 1995;50(9):688-97.

20. Alvarez F, Faundes A, Brache V, Tejada AS, Segal S. Prospective study of pituitary-ovarian function after tubal sterilization by the Pomeroy or Uchida techniques. Fertil Steril. 1989;51(4):604-8.

21. Pinho Neto JSL, Pinho Sales FS. Anticoncepção cirúrgica voluntária feminina. In: Oliveira HC, Lengruber I, editores. Tratado de ginecologia FEBRASGO. Rio de Janeiro: Revinter; 2000. v. 1, p. 470-5.

22. Cevrioglu AS, Degirmenci B, Acar M, Yilmazer M, Erol D, Kahraman A, et al. Examination of changes caused by tubal sterilization in ovarian hormone secretion and uterine and ovarian artery blood flow rates. Contraception. 2004;70(6):467-73.

23. Barros Leal JW, Câmara PAD. Ligadura tubária. In: Halbe HW, editor. Tratado de ginecologia. São Paulo: Roca; 2000. p. 873- 83.

24. Carmona F, Cristobal P, Casamitjana R, Balasch J. Effect of tubal sterilization on ovarian follicular reserve and function. Am J Obstet Gynecol. 2003; 189(2):447-52.

25. Fagundes ML, Mendes MC, Patta MC, Rodrigues R, Berezowski AT, de Moura MD, et al. Hormonal assessment of women submitted to tubal ligation. Contraception. 2005;71(4):309-14. 